\title{
Is Piecemeal Endoscopic Resection Acceptable for Early Colorectal Cancers in Certain Situations? A Single-Center French Study
}

\author{
Jean-Philippe Ratone ${ }^{1}$, Fabrice Caillol ${ }^{1}$, Christophe Zemmour ${ }^{2}$, Erwan Bories ${ }^{1}$, Christian Pesenti ${ }^{1}$, Sébastien Godat ${ }^{1}$, \\ Solene Hoibian ${ }^{1}$, Cécile De Chaisemartin ${ }^{3}$, Hélène Meillat ${ }^{3}$, Bernard Lelong ${ }^{3}$, Flora Poizat ${ }^{4}$, Marc Giovannini ${ }^{1}$
}

1) Paoli-Calmettes Institute,

Endoscopy Unit, Marseille;

2) Paoli-Calmettes Institute, Dept. Clin Res \& Invest, Biostat \& Methodolo Unit, Aix Marseille University, INSERM, IRD, SESSTIM, Marseille;

3) Paoli-Calmettes Institute, Surgery Unit, Marseille; 4) Paoli-Calmette Institute, Pathology Unit, Marseille, France

\author{
Address for correspondence: \\ Dr. Ratone Jean-Philippe \\ Paoli-Calmettes Institute, \\ Endoscopy Unit, Marseille, \\ France \\ jpratone@hotmail.fr
}

Received: 23.03 .2020

Accepted: 18.05 .2020

\begin{abstract}
Background \& Aims: The use of endoscopic treatment for early colorectal cancer (ECC) is increasing. The European guidelines suggest performing piecemeal endoscopic resection (pmR) for benign lesions and en bloc resection for ECC, especially for patients with favorable lymph node involvement risk evaluations. However, en bloc resections for lesions larger than two centimeters require invasive endoscopic techniques. Our retrospective single-center study aimed to determine the clinical impact of performing pmR for ECC rather than traditional en bloc resection.

Methods: A single-center study was performed between January 2012 and September 2017. All ECC patients were included. The main objective was to evaluate the number of patients who potentially underwent unnecessary surgery due to piecemeal resection. The secondary endpoints were as follows: disease-free survival (DFS), defined as the time from pmR to endoscopic failure (local recurrence not treatable by endoscopy), complication rate, number of patients who did not undergo surgery by default, and factors predictive of outcomes and complications.

Results: One hundred and forty-six ECC endoscopically treated patients were included. In total, 85 patients were excluded ( 71 who underwent en bloc resection, 14 with pending follow-up). Data from 61 patients (33 women and $28 \mathrm{men}$ ) were analyzed. Two patients underwent potentially unnecessary surgery [3.28\% (0.9\%$11.2 \%)$ ]. The DFS rate was $87 \%$ (75\%-93\%) at 6 months and $85 \%$ [72\%-92\%] at 12 months. The median followup time was 16.5 months (12.4-20.9). Three patients (4.9\%) had complications. One patient did not undergo surgery by default. A Paris classification of $0-2 c(H R=9.3(2.4-35.9), p<0.001)$ and Vienna classification of 5 $[\mathrm{HR}=16.3(3.3-80.4), \mathrm{p}<0.001]$ were factors associated with poor DFS.

Conclusion: Performing pmR in place of en bloc resection for ECC had a limited impact on patients. If the pathology (especially deep margins) is analyzable, careful monitoring could be acceptable in ECC patients who undergo pmR.
\end{abstract}

Key words: piecemeal endoscopic resection - early colorectal cancer.

Abbreviations: CRC: colorectal cancer; C1: midterm colonoscopy follow-up; C2: C1: midterm colonoscopy follow-up; C2: long-term colonoscopy; DFS: disease-free survival; ECC: early colorectal cancer; EMR: endoscopic mucosal resection; ESD: endoscopic submucosal dissection; LRR: locoregional recurrence; pmR: piecemeal endoscopic resection; pTis: intramucosal adenocarcinomas; pT1: adenocarcinomas with microinfiltration; pT1sm1 or ADKsm1: adenocarcinomas infiltration less than 1000 microns into the submucosa; R1: pathologically positive margins; UICC: International Union Against Cancer.

\section{INTRODUCTION}

In recent decades, the management of colorectal cancer (CRC) has improved both diagnostically and therapeutically. Despite this progress, CRC is the third most frequent cancer and has the fourth highest cancer mortality; thus, it remains a major public health problem worldwide [1].

The value of colonoscopy in the fight against CRC has been shown, as this technique can significantly reduce the mortality associated with CRC through screening and resection of preneoplastic lesions [2,3]. Starting in the 1990s, the possibility of resecting adenomas has increased with the introduction of endoscopic mucosal resection (EMR). This technique in which the main principle is based on the injection of the submucosa 
prior to polypectomy, has been further developed in Japan and around the world [4]. Some lesions, including both adenomas and superficial adenocarcinomas, have proven to be difficult to resect (size greater than $20 \mathrm{~mm}$, difficult position behind the folds, etc.) [5]. As the expertise of endoscopists and endoscopic technology improves, extensive lesions and flat lesions, including both adenomas and superficial adenocarcinomas, have been able to be resected [6]. Moreover, new techniques derived from EMR, such as piecemeal EMR (pmR) [7], endoscopic submucosal dissection (ESD) $[8,9]$ and a hybrid between the two techniques (hybrid ESD), have been developed [10]. This evolution has allowed colonoscopy to become not only a screening procedure but also a therapeutic technique. Apart from technical considerations, the main limitation of therapeutic endoscopy for superficial adenocarcinomas is correctly assessing the risk of lymph node invasion.

Several studies have defined the following subgroup of tumors for which the risk of lymph node involvement is considered to be zero or negligible: intramucosal adenocarcinomas [pTis according to the International Union Against Cancer (UICC) TNM classification [11] or a modified Vienna classification of 4.4] $[12,13]$ and any adenocarcinomas with microinfiltration (any pT1 tumor according to the UICC TNM classification or any tumors with a modified Vienna classification of 5), which is defined as tumors with a pT1 classification or modified Vienna classification of 5 and infiltration less than 1000 microns into the submucosa (pT1sm1 or ADKsm1) with certain qualitative criteria (welldifferentiated, no lymphovascular emboli, budding grade below 2 or 3) [14-16].

The management of colorectal adenomas has been the subject of several studies, the results of which have made it possible to draft European guidelines that perfectly outline the use of endoscopy in Western countries [16]. However, most studies included both colorectal adenomas and early colorectal cancers (ECCs) [7, 17]. Few studies have differentiated between adenomas with low-grade dysplasia and adenomas with highgrade dysplasia. The definition of ECC is not standardized; in Western countries, infiltrative lesions, or pT1 lesions, are sometimes considered early cancer, while in Japan, the term early cancer usually includes pTis and pT1 lesions $[18,19]$. In addition, the management strategies remain significantly different, with a so-called „selective ESD” strategy in Western countries and a strategy that strongly favors en bloc resection as soon as the lesion is inaccessible by en bloc EMR in Eastern Asia $[20,21]$. The strategy in Western countries reserves pmR for benign lesions or intramucosal adenocarcinomas without any identifiable risk of microinfiltration.

The use of pmR is often criticized for its increased risk of recurrence and because the pathology is difficult to interpret; therefore, predicting the risk of lymph node invasion is impossible, which is why ESD is recommended for lesions with suspected invasion into the upper layer of the submucosa. Although pretreatment endoscopic evaluation has greatly improved, the method is still imperfect, and $\mathrm{pmR}$ is sometimes performed for $\mathrm{pT} 1 \mathrm{sm} 1$ or deeper lesions, even in expert centers. Piecemeal resections are sometimes performed due to technical difficulties, particularly in terms of colon location.
In our practice, analyzing the deep tumor margins seems reliable for assessing the risk of lymph node invasion for cases of $\mathrm{pmR}$, and the use of $\mathrm{pmR}$ seems acceptable for lesions with acceptable deep tumor margins, even in the presence of ECC.

Herein, we report the results from our Western Interventional Endoscopy Center regarding the treatment of early CRCs over five years (from January 2012 to September 2017); en bloc resections were excluded.

The main aim of our study was to evaluate the number of patients who underwent potentially unnecessary surgery, as endoscopic resection was sufficient. The secondary outcomes were disease-free survival (DFS), remission rate after endoscopic treatment at the midterm (3-6 months) and long-term (> 12 months) follow-up visits, complication rate, recurrence management, number of patients who did not undergo surgery by default and the predictive factors for a poor prognosis.

\section{METHODS}

\section{Study design and data collection}

A single-center retrospective study was performed using data from all patients who underwent endoscopic resection for ECC between January 2012 and September 2017. Approval was obtained from the institutional review board and the local medical Ethics Committee. All patients were older than 18 years and had lesions considered pTis or pT1 according to the UICC TNM classification 2017 or a modified Vienna classification of 4.4 or 5 . All patients were identified using the full text software ConSoRe ${ }^{\mathrm{m} x}$. ConSoRe ${ }^{\mathrm{mm}}$ is a new generation of Big Data health software developed by Unicancer, one of Europe's largest cancer research organizations. ConSoRe ${ }^{\mathrm{Tm}}$ employs artificial intelligence based on machine learning and natural language processing.

All data were collected from the patients' electronic medical files, which contained information on medical consultations, medical observations, endoscopic procedures, and definitive histology; phone calls were conducted in cases of missing information. Patients who underwent en bloc resection, with failed endoscopic procedures or who were pending follow-up were excluded from the study.

We reported the following demographic and clinicopathological characteristics of the patients and lesions: gender, date of birth, lesion size, Paris classification, location of the lesion, Kikuchi classification, modified Vienna classification, qualitative pathology results (differentiation, presence of vascular or lymphatic emboli, presence of grade 2 or 3 budding), tumor margin, complication rate, and remission rates at the first clinical visit after endoscopic treatment (3 to 6 months) and at the second clinical visit after endoscopic treatment ( $>12$ months).

These data allowed us to evaluate our main objective as well as the secondary endpoints.

The main objective was to evaluate the number of patients who underwent potentially unnecessary surgery due to $\mathrm{pmR}$. Patients in this group underwent colorectal surgery for the following reasons: unclear pathology, intramucosal adenocarcinoma (pTis) with pathologically positive margins (R1) and definitive ypT0N0 pathology; pT1sm1R1 lesions 
with favorable qualitative features (well-differentiated, no vascular or lymphatic emboli, budding $<2$ ) and definitive ypT0N0 pathology; and local recurrence and failed second endoscopic resection. We hypothesized that for patients with local recurrence and a failed second endoscopic resection, an en bloc ESD could have prevented surgery by either facilitating a better evaluation of the resection margins or avoiding local recurrence from lesions that could not be treated endoscopically.

For pmR the secondary endpoints included the following: DFS, defined as the time from endoscopic resection to endoscopic failure (local recurrence not treatable by endoscopy); numbers of patients in remission at the midterm colonoscopy ( $\mathrm{C} 1=3-6$ months) and long-term colonoscopy ( $\mathrm{C} 2=12$ months) follow-up visits; complication rate; number of patients who did not undergo surgery by default; and the predictive factors of surgical outcomes and complications. To determine the number of patients who did not undergo surgery by default, we included patients with initially favorable histology and lymph node or distant metastatic evolution. For these patients, we hypothesized that the first pathology was erroneous due to $\mathrm{pmR}$.

\section{Procedures}

All procedures were performed under sedation with propofol or under general anesthesia with orotracheal intubation under the direct control of an anesthesiologist. The bowel was prepared with polyethylene glycol with ascorbic acid or sodium picosulfate. A PENTAX MEDICAL ${ }^{\mathrm{ma}}$ colonoscope (EC38-i10F2) or a PENTAX MEDICAL pediatric colonoscope (EC3490TFi) was used. $\mathrm{CO}_{2}$ insufflation was systematically used. Before any resection, all lesions were analyzed according to the Paris classification, and the pit pattern was analyzed using elements from the Kudo, Sano, NICE or JNET classifications (without zoom and using i-scan PENTAX MEDICAL ${ }^{\mathrm{m}}$ instead NBI OLYMPUS ${ }^{\mathrm{m}}$ ) [22-25]. In cases of 0-IIc lesions or a moderately modified vascular pit pattern without an avascular zone, piecemeal resection was performed at the operator's discretion if the lifting sign was positive.

Endoscopic ultrasonography was performed in a few cases of difficult rectal lesions, the appearance of which suggested the presence of an infiltrative lesion (>T1); however, endoscopic ultrasonography was not performed routinely.

Piecemeal endoscopic resection was performed after injecting saline solution or viscous solution. Indigo carmine was used as the inner dye to facilitate visualization of the lesion margins and identification of the correct plane for resection. During this period, a 25-mm COOK MEDICAL ${ }^{\mathrm{Tm}}$ snare, a hexagonal COOK MEDICAL ${ }^{\mathrm{mm}}$ snare, and a monofil ${ }^{\mathrm{Tm}}$ ultrasnare (10 and $15 \mathrm{~mm}$ ) MEDWORK ${ }^{\mathrm{mm}}$ (Höchstadt, Germany) were used. All resections were performed according to the classical technique of pmR while avoiding mucosal bridges. In a few cases, hybrid ESD was performed with a Dual knife ${ }^{\text {Tw }}$ or ITknife nano $^{\mathrm{Tm}}$ (OLYMPUS ${ }^{\mathrm{Tm}}$, Tokyo, Japan), but no en bloc resections were performed. During the procedure, special attention was paid to remove the residual adenomatous tissue and to check the lateral margins. Preventive hemostasis was also performed at the end of the resection.
Ambulatory care or a short hospitalization stay was decided by the responsible operator and the anesthesiologist according to the patient's comorbidities.

All operators had at least 3 years of experience in $\mathrm{pmR}$ and ESD.

Endoscopic recurrence was defined by typical adenomatous or tumoral tissue confirmed on biopsies or on the second endoscopic resection pathology.

\section{Statistical analysis}

All statistical analyses were performed with a significance level of $\alpha=0.05$ and with $\mathrm{SAS}^{\otimes} 9.3$ software. The data were summarized by using counts (frequencies) for qualitative variables and means (standard deviations) and medians (min$\max$ ) for quantitative variables. The number of patients who underwent potentially unnecessary surgery due to piecemeal resection was estimated, along with the Wilson bilateral confidence interval. Disease-free survival was estimated by using the Kaplan-Meier method. Patients without endoscopic failure were right-censored at the date of their last follow-up. The 6- and 12-month DFS rates were estimated, along with their bilateral confidence intervals. The impact of each factor (Paris classification of $0-2 \mathrm{c}$ vs other, location of the lesion, tumor size $>4 \mathrm{~cm}$ vs $\leq 4 \mathrm{~cm}$, modified Vienna classification of 5 vs 4.4 ) on DFS was assessed by log-rank tests. The associated hazard ratios (HRs) were estimated along with the corresponding bilateral Wald confidence intervals. The follow-up period was estimated by using the inverse Kaplan-Meier method.

\section{Reason for the exclusion of en bloc resection}

En bloc resection (ESD and EMR) was excluded because the groups were not comparable (more rectum involvement in the en bloc group; smaller diameter and more difficult locations in the pmR group). Moreover, aim of this study was not to compare ESD and EMR but evaluate pmR in cases of ECC.

\section{RESULTS}

\section{Patient and lesion characteristics}

One hundred forty-six ECC patients (78 men and 68 women) treated by endoscopy were identified using the ConSoRe $^{\text {th }}$ full text search software in our center (PaoliCalmettes Institute, France). After the first analysis, 71 patients were excluded because they underwent en bloc resection (ESD, hybrid ESD or EMR), and 14 patients were excluded because they were pending follow-up. In total, the data from 61 ECC patients ( 33 women and 28 men) treated by pmR was analyzed. Each of the 61 patients had a single lesion that met the study criteria. The median age was 66 years old (range: $39-88$ ). The average lesion size was $36.15 \mathrm{~mm}$. The lesions were located in the right colon $(21 / 61,34.4 \%)$, transverse colon $(7 / 61$, $11.5 \%)$, left colon $(14 / 61,23 \%)$ and rectum $(19 / 61,31.1 \%)$. Forty-eight patients had pTis lesions (UICC classification) (4.4 modified Vienna classification). Thirteen patients had pT1 lesions according to UICC classification and a classification of 5 according to the modified Vienna classification (one pT1sm1 and twelve pT $1 \mathrm{sm} 2$ lesions). Fifty-one lesions were resected by classical pmR, and 10 lesions were treated by pmR using ESD 
instruments. All patient and lesion characteristics are shown in Table I. The study design is shown in Fig. 1.

Table I. Patient and lesion characteristics

\begin{tabular}{|c|c|}
\hline \multicolumn{2}{|l|}{ Gender } \\
\hline Male & $28(45.9 \%)$ \\
\hline Age median (min-max) & $66(29-88)$ \\
\hline \multicolumn{2}{|l|}{ Paris classification } \\
\hline Other & $55(90.2 \%)$ \\
\hline $0-2 c$ & $6(9.8 \%)$ \\
\hline \multicolumn{2}{|l|}{ Tumor diameter (mm) } \\
\hline Mean (SD) & $36.2(10.5)$ \\
\hline$(0-20)$ & $4(6.6 \%)$ \\
\hline$(21-40)$ & $46(75.4 \%)$ \\
\hline$>40$ & $11(18 \%)$ \\
\hline \multicolumn{2}{|l|}{ Location } \\
\hline Right colon & $21(34.4 \%)$ \\
\hline Transverse colon & $7(11.5 \%)$ \\
\hline Left colon & $14(23 \%)$ \\
\hline Rectum & $19(31.1 \%)$ \\
\hline \multicolumn{2}{|l|}{ Resection type } \\
\hline cpmEMR & $51(83.6 \%)$ \\
\hline hpmEMR & $10(16.4 \%)$ \\
\hline \multicolumn{2}{|l|}{ Vienna classification } \\
\hline 4.4 & $48(78.7 \%)$ \\
\hline 5 & $13(21.3 \%)$ \\
\hline \multicolumn{2}{|l|}{ Kikuchi classification } \\
\hline ADKim & $48(78.7 \%)$ \\
\hline pT1sm1 & $2(3.3 \%)$ \\
\hline pT1sm2 & $11(18 \%)$ \\
\hline \multicolumn{2}{|l|}{ Budding grade 2-3 } \\
\hline No & $54(88.5 \%)$ \\
\hline Yes & $7(11.5 \%)$ \\
\hline \multicolumn{2}{|l|}{ Deep margins } \\
\hline Free & $57(93.4 \%)$ \\
\hline Pathologic & $4(6.6 \%)$ \\
\hline
\end{tabular}

cpmEMR: classical piecemeal endoscopic mucosal resection; hpmEMR: hybrid piecemeal endoscopic mucosal resection i.e., partial use of dissection instruments

\section{Main objective results}

We considered that 2 patients underwent potentially unnecessary surgery $(3.28 \%$ [0.9\%-11.2\%]) based on the criteria outlined in the material and methods. The first patient was a 70-year-old woman who underwent $\mathrm{pmR}$ for a right colonic lesion of $40 \mathrm{~mm}$. The pathologist concluded that the lesion was pTis with free deep tumor margins (R0). At C1, the recurrence could not be treated by endoscopy, and the patient was referred for oncological surgery. The second patient was a 55-year-old woman with a lesion of 50 millimeters in the rectum. She had an intramucosal adenocarcinoma (with free deep tumor margins; R0) and underwent $\mathrm{R} 0$ resection. At C1, she presented with recurrence, and the second endoscopic resection failed. None of the other patients met the other criteria of the primary objective (unclear pathology, intramucosal adenocarcinoma $\mathrm{R} 1$ or $\mathrm{pT} 1 \mathrm{sm} 1 \mathrm{R} 1$ resection with favorable qualitative features). These results are shown in Table II.

\section{Secondary endpoint results}

The DFS rate was $87 \%(75 \%-93 \%)$ at 6 months and $85 \%$ (72\%-92\%) at 12 months. Specifically, no mortality events occurred after 9 months. The median follow-up time was 16.5 months (12.4-20.9). The DFS results are shown in Fig. 2.

Eight patients were referred before $\mathrm{C} 1$ for colorectal surgery based on their histology results. Forty-four patients (72.1\%) were in remission at $\mathrm{C} 1$, and nine patients had recurrence at C1. These results are shown in figure 1 and Table II.

Thirty-five of the 45 patients who were not lost to followup $(77.8 \%)$ were in remission at C2. Seven out of 9 patients with locoregional recurrence (LRR) were successfully treated by a second endoscopic resection, and $2 / 9$ patients with LRL needed oncologic surgery. All patients with good disease control at $\mathrm{C} 1$ had no recurrence at $\mathrm{C} 2$. These results are shown in Fig. 1 and Table II. Finally, at the last follow-up, 51 patients $(83.6 \%)$ had achieved remission at least once after receiving endoscopic treatment.

The median hospital length of stay was 2 days (range: 1-7), and the mean was 2.4 days (0.9). These results are shown in Table II.

Three patients (4.9\%) developed complications, including two perforations and one case of postoperative bleeding. All complications were endoscopically managed without the need for a transfer to the intensive care unit. There were no deaths related to endoscopic resection. These results are shown in Table II.

During the follow-up period, there were 9 patients with LRR. Seven patients were successfully treated by a second endoscopic procedure (with a satisfactory endoscopic check 3 or 6 months later). Two patients underwent classical oncologic colectomy. The first patient had a ypPT1N0 lesion, and the second had a ypT0N1 lesion. These results are shown in Table II. Example of recurrence before and after second endoscopic procedure is shown in Fig. 3.

One patient did not undergo surgery by default. This patient had an intramucosal adenocarcinoma with cancer cell-free deep margins at the first piecemeal resection, but he had recurrence with pathologic tumor margins treated by a second endoscopic procedure. The definitive histology indicated ypT0N1. We believed that the initial margin analysis evaluation was poorly conducted, which was potentially due to the use of piecemeal resection. These results are shown in Table II.

A Paris classification of $0-2 \mathrm{c}[\mathrm{HR}=9.3(2.4-35.9), \mathrm{p}<0.001]$ and a Vienna classification of $5[\mathrm{HR}=16.3(3.3-80.4), \mathrm{p}<0.001]$ were the only factors significantly associated with poor DFS. No differences were found for the other two criteria (tumor size $>4 \mathrm{~cm}$ vs tumor size $\leq 4 \mathrm{~cm}$; rectal vs colon location). These results were determined from univariate analyses and are shown in Table III

\section{DISCUSSION}

Regarding the main objective, the negative impact of performing a pmR instead of an en bloc resection seems to 

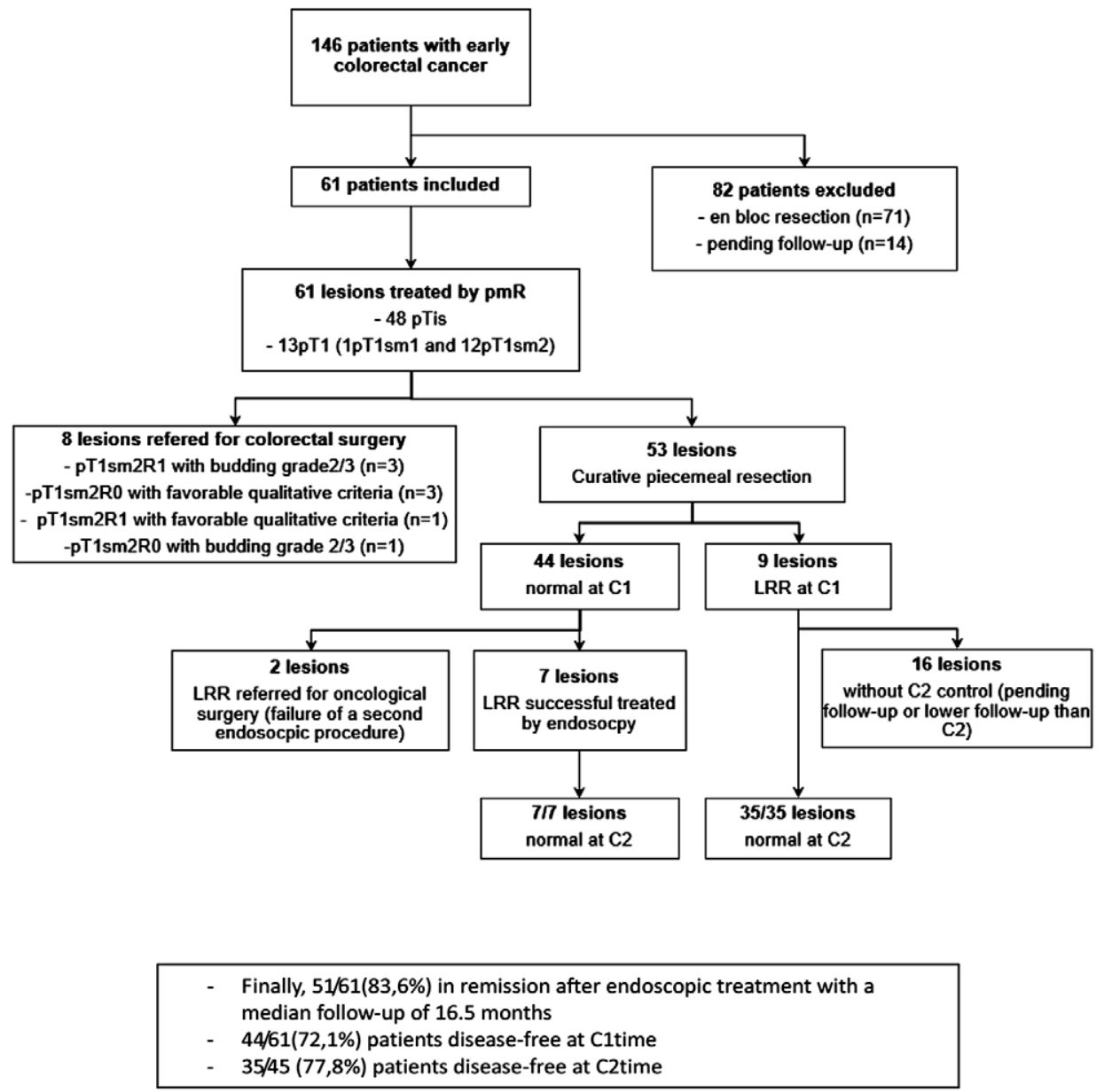

Fig. 1. Study design and main results. pmR: piecemeal endoscopic resection; LRR: local regional recurrence; $\mathrm{C} 1$ : endoscopic control at midterm (3-6 months); $\mathrm{C} 2=$ endoscopic control at long-term (>12 months)

be small, as only two patients (3.3\%) underwent unnecessary surgery. As a result, unlike in previous studies, the pathology determined in this tertiary center by $\mathrm{pmR}$ was interpretable, which is the main take-home message of our series [26]. Both of these patients had clinical recurrence that could not be treated endoscopically.

Clearly, this result implies that ESD would have been possible; however, we cannot definitively state this conclusion. Indeed, ESD is less developed in Western countries than in Asian countries due to its delayed introduction, the required learning curve and differences in the organization of interventional endoscopy units [27-32]. In a meta-analysis, the $\mathrm{R} 0$ resection rate was $71.3 \%$ in non-Asian countries versus $85.6 \%$ in Asian countries, although the incidence of adverse events that required surgery was $3.1 \%$ versus $0.8 \%$, respectively [27]. These results allowed the European guidelines to continue recommending $\mathrm{pmR}$ as a reference treatment for noninvasive lesions [16], although some recent studies have suggested that the differences between Eastern and Western countries have been reduced $[33,34]$. Regarding the secondary objective of patients who did undergo surgery by default, only one patient met the criteria. We believe that this patient would have benefited from a better histological analysis with en bloc treatment than with pmR and therefore would have undergone a more radical surgery after the initial endoscopic resection. Moreover, together with the pathology team, we have voluntarily established broad criteria that include the main objective of unnecessary surgery and the secondary criterion of not undergoing surgery by default. Indeed, these two criteria evaluate the clinical impact of performing piecemeal resection in ECC patients, although performing en bloc resection is desirable.

Interestingly, even in cases of degenerated lesions and pmR, the results were excellent $(83.6 \%$ of patients achieved remission) and indicated low morbidity (4.9\%). In addition, no complications that required surgery or transfer to an intensive care unit occurred. Of the 53 patients who underwent curative resection, 9 had LRR at C1 (17\%). Recurrence could be treated endoscopically in $7 / 9$ patients $(77.7 \%)$. These results are similar to another interesting series involving performing $\mathrm{pmR}$ for most adenomas. Moss et al. [7] described a recurrence rate of $16 \%$, and $94.5 \%$ of the recurrences were treated endoscopically.

Predictably, microinfiltration and ulceration (Paris classification of $0-2 c$ ) were predictive factors for failed endoscopic treatment of ECC. Surprisingly, a tumor size $>4$ $\mathrm{cm}$ was not a predictive factor of poor efficacy, even though this factor was expected to be a predictor [7]. The duration of hospitalization was acceptable (median of 2 days), and the organ preservation rate was excellent. It is likely but uncertain that en bloc ESD has a higher organ preservation rate than 
Table II. Main results

\begin{tabular}{|c|c|}
\hline Event & Statistics \\
\hline \multicolumn{2}{|c|}{ Remission after endoscopic treatment } \\
\hline Yes & $51(83.6 \%)$ \\
\hline No & $10(12.4 \%)$ \\
\hline \multicolumn{2}{|l|}{ Remission at $\mathrm{C} 1$} \\
\hline Yes & $44(72.1 \%)$ \\
\hline No & $17(27.9 \%)$ \\
\hline \multicolumn{2}{|l|}{ Remission rate at $\mathrm{C} 2$} \\
\hline Yes & $35(77.8 \%)$ \\
\hline No & $10(22.2 \%)$ \\
\hline \multicolumn{2}{|l|}{ Hospital stay time (days) } \\
\hline Mean (SD) & $2.4(0.9)$ \\
\hline Median (Min-Max) & $2(1-7)$ \\
\hline \multicolumn{2}{|l|}{ LRR treatment } \\
\hline Surgery & $2(22.2 \%)$ \\
\hline Endoscopy & $7(77.8 \%)$ \\
\hline \multicolumn{2}{|l|}{ Unnecessary surgery } \\
\hline \multicolumn{2}{|l|}{ Not operated by default } \\
\hline No & $60(98.4 \%)$ \\
\hline Yes & $1(1.6 \%)$ \\
\hline Adverse events & $60(98.4 \%)$ \\
\hline No & $3(4.9 \%)$ \\
\hline Yes & $58(95.1 \%)$ \\
\hline \multicolumn{2}{|l|}{ Adverse event types } \\
\hline Post procedural bleeding & $1(33.3 \%)$ \\
\hline Perforation & $2(66.6 \%)$ \\
\hline \multicolumn{2}{|l|}{ Complication management } \\
\hline Endoscopic & $3(100 \%)$ \\
\hline Surgery or other & $0(0 \%)$ \\
\hline
\end{tabular}

LRR=locoregional relapse

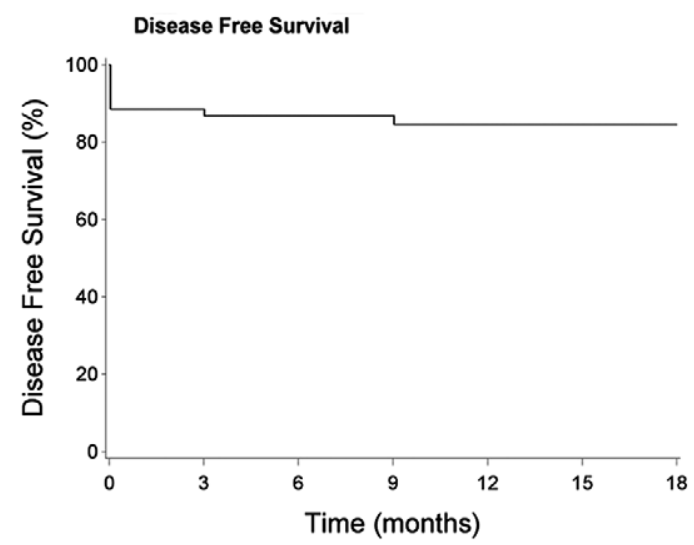

Fig. 2. Disease-free survival (DFS) estimate by the Kaplan-Meier method.

piecemeal surgery because the incidence of perforations that require surgery is rarely zero in Western series, although the rate has been increasing $[27,35]$.

In our study, we voluntarily decided not to compare the group "piecemeal" and the group "en bloc" because they were not comparable (size and location).
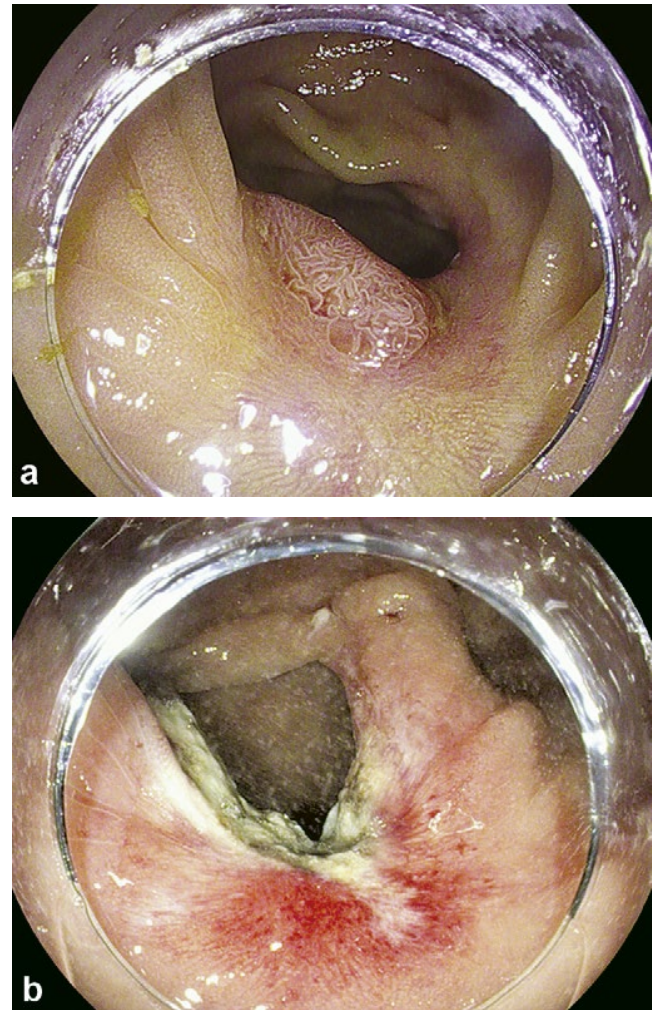

Fig. 3. Example of recurrence before (2a) and after (2b) the second endoscopic procedure

Table III. Predictive factors of poor disease-free survival prognosis

\begin{tabular}{lcc}
\hline Contrast & $\begin{array}{c}\text { Hazard Ratio } \\
(95 \% \mathrm{CI})\end{array}$ & p-value \\
\hline Paris classification 0 -2c vs Other & $9.3(2.4-35.9)$ & 0.001 \\
$\begin{array}{l}\text { Location of the lesion, Rectum vs } \\
\text { Colon }\end{array}$ & $0.6(0.1-3.0)$ & 0.55 \\
Tumor size $>4$ cm vs $\leq 4 \mathrm{~cm}$ & $1.3(0.3-6.3)$ & 0.74 \\
Vienne classification 5 vs 4.4 & $16.3(3.3-80.4)$ & $<0.001$ \\
\hline
\end{tabular}

This study has several limitations, in addition to its retrospective nature. Several factors counter the recommendation of a systematic pmR. The patient who did not undergo surgery by default for a tumor initially classified as 4.4 with the Vienna classification presented a definitive ypT0N1 histology; this result clearly suggests an initial underestimation during endoscopic resection, which is probably because of the fragmented nature of resection. In contrast, for the 2 patients who underwent unnecessary surgery, the extra treatment seems acceptable at the oncological level; however, the absence of organ preservation can be harmful in the long run. Clearly, another limitation at the medico-economic level is the cost of repeated colonoscopies.

The excellent overall survival rate of ECC patients in our study $(100 \%)$ was similar to that in the literature $[19,36]$ and should motivate us to treat as conservatively as possible to achieve an extremely low rate of complications, but patients who did not undergo surgery by default can experience severe oncological consequences.

This single-center study emphasizes the important role of endoscopy in managing ECC. In addition, the identification 
of endoscopically resectable pT1 and p Tis lesions is improving with the development of screening and therapeutic endoscopic procedures [37]. Endoscopic submucosal resection is still a difficult technique to perform and is reserved for some tertiary centers; the use of ESD to treat all early cancers in Europe, especially those in difficult locations (right colon, between two folds, etc.), still seems implausible. Surgery, an alternative treatment choice, must be reserved for patients with a significant risk of lymph node invasion due to the significant risk of morbidity and mortality (6\%) [38].

A precise preoperative analysis of superficial colorectal lesions before resection is absolutely necessary using the Paris classification and pit pattern analysis derived from the Kudo, SANO, JNET or NICE classifications [22-25]. Although the accuracy is imperfect (78-92\%), they allowed the selection of good candidates for endoscopic resection [39]. While endoscopic ultrasound is often limited for distinguishing between superficial and deep invasion of submucosa, it would be a supportive tool for identifying deep-massive submucosal invasion in the suspected cases.

Our results show that in cases of $0-2 \mathrm{c}$ lesions or changes in the minimal modified vascular pit pattern (Sano equivalent IIIa, JNET equivalent $2 \mathrm{~B}$ ) and if the lifting sign is positive, $\mathrm{pmR}$ is an efficient and safe technique. On the one hand, in these situations, we believe pmR is the best choice when ESD is not reasonable (due to the location, size etc.) or the patient has comorbidities that makes a colectomy a high-risk surgery. On the other hand, $0-2 \mathrm{c}$ lesions without a negative lifting sign or a completely avascular pit pattern is a formal contraindication for pmR.

Nevertheless, we need a new classification that is more sensible and reproducible and better adapted for use by all expert endoscopists (Western and Eastern), with all scopes for all types of lesions (adenomatous and serrated) [40].

Ultimately, our study agrees with the European guidelines, which suggest that for suspected superficial degenerated colorectal lesions, en bloc resection is the preferred method over pmR to allow for a more reliable pathological analysis as well as to prevent patient monitoring [16]. However, when piecemeal resection has been performed due to a poor initial assessment or technical failure and if the deep tumor margins are analyzable, careful monitoring is quite acceptable due to the small long-term clinical impact on patient outcomes. No disease-related deaths occurred in this study.

\section{CONCLUSIONS}

Performing pmR when en bloc resection appeared more desirable had a real but limited impact on our patients. We hope that in the future, the improved characterization of colorectal lesions (perhaps with the help of artificial intelligence) as well as the development of advanced ESD training programs in Western endoscopy centers will help to further optimize these results for our patients.

Conflicts of interest: M.G. is a member of the advisory board for COOK MEDICAL ${ }^{\mathrm{Tw}}$. All the others authors declare no conflict of interests.
Authors' contributions: J.P.R.: principal investigator and endoscopy operator, conceived the study and drafted the manuscript; F.C.: second investigator and endoscopy operator, conceived the study. C.Z. statistical analysis. E.B.,C.P., S.G., S.H: endoscopy operators, C.D.C, H.M. and B.L: colorectal surgeons. F.P.: main pathologist. M.G.: supervised the study and critically revised the paper. All the authors approved the final version of the manuscript.

\section{REFERENCES}

1. Ferlay J, Shin HR, Bray F, Forman D, Mathers C, Parkin DM. Estimates of worldwide burden of cancer in 2008: GLOBOCAN 2008. Int J Cancer 2010;127:2893-2917. doi:10.1002/ijc.25516

2. Zauber AG, Winawer SJ, O'Brien MJ, et al. Colonoscopic polypectomy and long-term prevention of colorectal-cancer deaths. N Engl J Med 2012;366:687-696. doi:10.1056/NEJMoa1100370

3. Nishihara R, Wu K, Lochhead P, et al. Long-term colorectal-cancer incidence and mortality after lower endoscopy. N Engl J Med 2013;369:1095-1105. doi:10.1056/NEJMoa1301969

4. Shirai M, Nakamura T, Matsuura A, Ito Y, Kobayashi S. Safer colonoscopic polypectomy with local submucosal injection of hypertonic saline-epinephrine solution. Am J Gastroenterol 1994;89:334-338.

5. Gallegos-Orozco JF, Gurudu SR. Complex colon polypectomy. Gastroenterol Hepatol (N Y) 2010;6:375-382.

6. Lee TJ, Rees CJ, Nickerson C, et al. Management of complex colonic polyps in the English Bowel Cancer Screening Programme. Br J Surg 2013;100:1633-16339. doi:10.1002/bjs.9282

7. Moss A, Williams SJ, Hourigan LF, et al. Long-term adenoma recurrence following wide-field endoscopic mucosal resection (WF-EMR) for advanced colonic mucosal neoplasia is infrequent: results and risk factors in 1000 cases from the Australian Colonic EMR (ACE) study. Gut 2015;64:57-65. doi:10.1136/gutjnl-2013-305516

8. Repici A, Hassan C, Pagano N, et al. High efficacy of endoscopic submucosal dissection for rectal laterally spreading tumors larger than $3 \mathrm{~cm}$. Gastrointest Endosc 2013;77:96-101. doi:10.1016/j. gie.2012.08.036

9. Probst A, Golger D, Anthuber M, Markl B, Messmann H. Endoscopic submucosal dissection in large sessile lesions of the rectosigmoid: learning curve in a European center. Endoscopy 2012;44:660-667. doi:10.1055/s-0032-1309403

10. Bae JH, Yang DH, Lee S, et al. Optimized hybrid endoscopic submucosal dissection for colorectal tumors: a randomized controlled trial. Gastrointest Endosc 2016;83:584-592. doi:10.1016/j.gie.2015.06.057

11. Amin MB, Greene FL, Edge SB, et al. The Eighth Edition AJCC Cancer Staging Manual: Continuing to build a bridge from a population-based to a more "personalized" approach to cancer staging. CA Cancer J Clin 2017;67:93-99. doi:10.3322/caac. 21388

12. Dixon MF. Gastrointestinal epithelial neoplasia: Vienna revisited. Gut 2002;51:130-131. doi:10.1136/gut.51.1.130

13. Schlemper RJ, Riddell RH, Kato Y, et al. The Vienna classification of gastrointestinal epithelial neoplasia. Gut 2000;47:251-255. doi:10.1136/ gut.47.2.251

14. Beaton C, Twine CP, Williams GL, Radcliffe AG. Systematic review and meta-analysis of histopathological factors influencing the risk of lymph node metastasis in early colorectal cancer. Colorectal Dis 2013;15:788797. doi:10.1111/codi.12129 
15. Wada H, Shiozawa M, Katayama K, et al. Systematic review and meta-analysis of histopathological predictive factors for lymph node metastasis in T1 colorectal cancer. J Gastroenterol 2015;50:727-734. doi:10.1007/s00535-015-1057-0

16. Ferlitsch M, Moss A, Hassan C, et al. Colorectal polypectomy and endoscopic mucosal resection (EMR): European Society of Gastrointestinal Endoscopy (ESGE) Clinical Guideline. Endoscopy 2017;49:270-297. doi:10.1055/s-0043-102569

17. Backes Y, Moons LMG, van Bergeijk JD, et al. Endoscopic mucosal resection (EMR) versus endoscopic submucosal dissection (ESD) for resection of large distal non-pedunculated colorectal adenomas (MATILDA-trial): rationale and design of a multicenter randomized clinical trial. BMC Gastroenterol 2016;16:56. doi:10.1186/s12876-016-0468-6

18. Bartel MJ, Brahmbhatt BS, Wallace MB. Management of colorectal T1 carcinoma treated by endoscopic resection from the Western perspective. Dig Endosc 2016;28:330-341. doi:10.1111/den.12598

19. Saitoh Y, Inaba Y, Sasaki T, Sugiyama R, Sukegawa R, Fujiya M. Management of colorectal T1 carcinoma treated by endoscopic resection. Dig Endosc 2016;28:324-329. doi:10.1111/den.12503

20. Bahin FF, Heitman SJ, Rasouli KN, et al. Wide-field endoscopic mucosal resection versus endoscopic submucosal dissection for laterally spreading colorectal lesions: a cost-effectiveness analysis. Gut 2018;67:1965-1973. doi:10.1136/gutjnl-2017-313823

21. Tanaka S, Kashida H, Saito Y, et al. JGES guidelines for colorectal endoscopic submucosal dissection/endoscopic mucosal resection. Dig Endosc 2015;27:417-434. doi:10.1111/den.12456

22. Sano Y, Tanaka S, Kudo SE, et al. Narrow-band imaging (NBI) magnifying endoscopic classification of colorectal tumors proposed by the Japan NBI Expert Team. Dig Endosc 2016;28:526-533. doi:10.1111/den.12644

23. Endoscopic Classification Review G. Update on the paris classification of superficial neoplastic lesions in the digestive tract. Endoscopy 2005;37:570-578. doi:10.1055/s-2005-861352

24. Hayashi N, Tanaka S, Hewett DG, et al. Endoscopic prediction of deep submucosal invasive carcinoma: validation of the narrow-band imaging international colorectal endoscopic (NICE) classification. Gastrointest Endosc 2013;78:625-632. doi:10.1016/j.gie.2013.04.185

25. Kudo S. Endoscopic mucosal resection of flat and depressed types of early colorectal cancer. Endoscopy 1993;25:455-461. doi:10.1055/s-2007-1010367

26. Pioche M, Rivory J, Jeremie J. Colorectal endoscopic submucosal dissection for all LSTs: histological information loss due to piecemeal EMR is no longer acceptable. Endosc Int Open 2019;7:E1195-E1196. doi:10.1055/a-0982-3223

27. Fuccio L, Hassan C, Ponchon T, et al. Clinical outcomes after endoscopic submucosal dissection for colorectal neoplasia: a systematic review and meta-analysis. Gastrointest Endosc 2017;86:74-86.e17. doi:10.1016/j. gie.2017.02.024

28. Fuccio L, Repici A, Hassan C, et al. Why attempt en bloc resection of non-pedunculated colorectal adenomas? A systematic review of the prevalence of superficial submucosal invasive cancer after endoscopic submucosal dissection. Gut 2018;67:1464-1474. doi:10.1136/ gutjnl-2017-315103

29. Heitman SJ, Tate DJ, Bourke MJ. Optimizing Resection of Large Colorectal Polyps. Curr Treat Options Gastroenterol 2017;15:213-229. doi:10.1007/s11938-017-0131-5

30. Heitman SJ, Bourke MJ. Endoscopic submucosal dissection and EMR for large colorectal polyps: "the perfect is the enemy of good". Gastrointest Endosc 2017;86:87-89. doi:10.1016/j.gie.2017.03.022

31. Saito Y, Kawano H, Takeuchi Y, et al. Current status of colorectal endoscopic submucosal dissection in Japan and other Asian countries: progressing towards technical standardization. Dig Endosc 2012;24 Suppl 1:67-72. doi:10.1111/j.1443-1661.2012.01282.x

32. Ma MX, Bourke MJ. Complications of endoscopic polypectomy, endoscopic mucosal resection and endoscopic submucosal dissection in the colon. Best Pract Res Clin Gastroenterol 2016;30:749-767. doi:10.1016/j.bpg.2016.09.009

33. Santos-Antunes J, Baldaque-Silva F, Marques M, Lopes J, Carneiro F, Macedo G. Real-life evaluation of the safety, efficacy and therapeutic outcomes of endoscopic submucosal dissection in a Western tertiary centre. United European Gastroenterol J 2018;6:702-709. doi:10.1177/2050640618755237

34. Milano RV, Viale E, Bartel MJ, Notaristefano C, Testoni PA. Resection outcomes and recurrence rates of endoscopic submucosal dissection (ESD) and hybrid ESD for colorectal tumors in a single Italian center. Surg Endosc 2018;32:2328-2339. doi:10.1007/s00464-017-5928-8

35. Daoud DC, Suter N, Durand M, Bouin M, Faulques B, von Renteln D. Comparing outcomes for endoscopic submucosal dissection between Eastern and Western countries: A systematic review and meta-analysis. World J Gastroenterol 2018;24:2518-2536. doi:10.3748/wjg.v24. i23.2518

36. Silva GL, de Moura EG, Bernardo WM, et al. Endoscopic versus surgical resection for early colorectal cancer-a systematic review and meta-analysis. J Gastrointest Oncol 2016;7:326-335. doi:10.21037/ jgo.2015.10.02

37. Tinmouth J, Lansdorp-Vogelaar I, Allison JE. Faecal immunochemical tests versus guaiac faecal occult blood tests: what clinicians and colorectal cancer screening programme organisers need to know. Gut 2015;64:1327-1337. doi:10.1136/gutjnl-2014-308074

38. Duraes LC, Stocchi L, Steele SR, et al. The Relationship Between Clavien-Dindo Morbidity Classification and Oncologic Outcomes After Colorectal Cancer Resection. Ann Surg Oncol 2018;25:188-196. doi:10.1245/s10434-017-6142-6

39. Pu LZC, Cheong KL, Koay DSC, et al. Randomised controlled trial comparing modified Sano's and narrow band imaging international colorectal endoscopic classifications for colorectal lesions. World J Gastrointest Endosc 2018;10:210-218. doi:10.4253/wjge.v10.i9.210

40. Fabritius M, Gonzalez JM, Becq A, et al. A simplified table using validated diagnostic criteria is effective to improve characterization of colorectal polyps: the CONECCT teaching program. Endosc Int Open 2019;7:E1197-E1206. doi:10.1055/a-0962-9737 